\title{
The Interaction Between Point Defects and Edge Dislocation in BCC Iron
}

\author{
Vijay Shastry
}

Tomas Diaz de la Rubia

This paper was prepared for submittal to the

Society of Engineering Science 35th Annual Technical Meeting

Pullman, WA

September 27-30, 1998

October 12, 1998

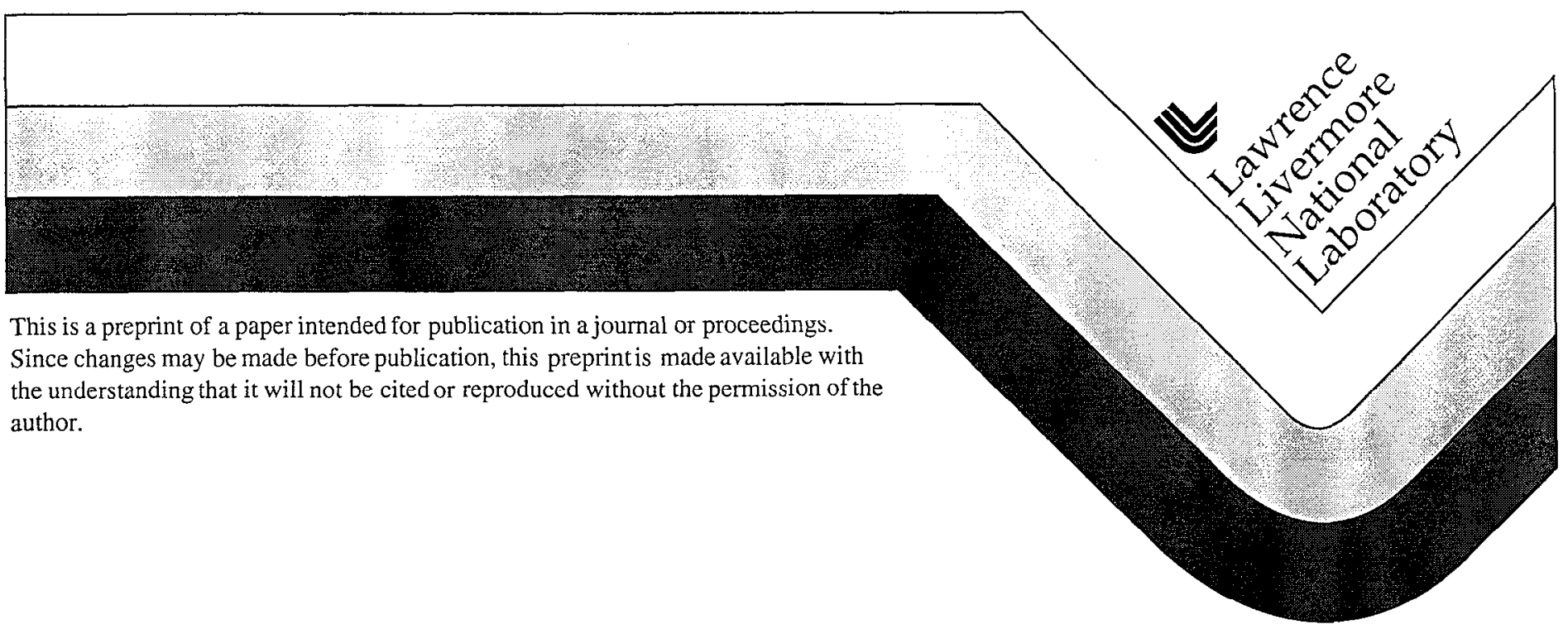




\section{DISCLAIMER}

This document was prepared as an account of work sponsored by an agency of the United States Government. Neither the United States Government nor the University of California nor any of their employees, makes any warranty, express or implied, or assumes any legal liability or responsibility for the accuracy, completeness, or usefulness of any information, apparatus, product, or process

disclosed, or represents that its use would not infringe privately owned rights. Reference herein to any specific commercial product, process, or service by trade name, trademark, manufacturer, or otherwise, does not necessarily constitute or imply its endorsement, recommendation, or favoring by the United States Government or the University of California. The views and opinions of authors expressed herein do not necessarily state or reflect those of the United States Government or the University of California, and shall not be used for advertising or product endorsement purposes. 


\title{
The interaction between point defects and edge dislocation in BCC iron*
}

\author{
Vijay Shastry and Tomas Diaz de la Rubia, \\ Chemistry and Materials Science Directorate, \\ Lawrence Livermore National Laboratory, \\ Livermore, CA 94551.
}

October 12, 1998

\begin{abstract}
We present results of atomistic simulations of the interaction between self interstitial atoms and vacancies with edge dislocations in BCC iron. The calculations are carried out using molecular dynamics with an energy minimization scheme based on the quasi-Newton approach and use the Finnis-Sinclair interatomic potential for BCC iron developed by Ackland et al. Large anisotropy in the strain field of self interstitials is observed and it causes strong interaction with edge dislocations even when the defect is located on the dislocation glide plane. For vacancies, the relaxation volume is smaller and much more isotropic, which results in a far weaker interaction with the dislocation. A temperature dependent capture radius for vacancies and self interstitials is extracted from the simulations. The difference between the capture radii of vacancies and self interstitials is used to define the sink strength of the dislocation. Large deviations are observed from the predictions of elasticity based on treating point defects as isotropic dilatational centers. Further, the capture radius of edge dislocations in BCC iron is observed to be small and is of the order of $1-3 \mathrm{~nm}$ for self interstitials.
\end{abstract}

\section{Introduction}

Irradiation of a material with energetic particles such a fission neutrons or heavy ions introduces a high super-saturation of point defect and defect clusters [1]. At sufficiently high temperature, some or all of these defects migrate and interact, thereby driving the system back toward equilibrium and possibly altering the chemistry and microstructure in the process. One of the most important aspects of microstructure evolution in irradiated materials is the nature and magnitude of the interaction bctwcen point defects and sinks such as dislocations $[1,2]$. In addition to this, the interaction between interstitial dislocation loops and

*This work has been conducted under the auspices of the U.S. Department of Energy by Lawrence Livermore National Laboratory under contract number W-7405-ENG-48. 
dislocations is also thought to be important in describing irradiation-induced hardening [3]. It has recently been argued that irradiation-hardening may indeed be strongly related to the production and one dimensional glide of the small self-interstitial clusters and details of subsequent interaction with the dislocation core [3].

Within the context of elasticity theory, the hydrostatic stress field of an edge dislocation interacts with the relaxation volume of the point defect [4]. This interaction lowers the free energy of the system thus reducing the defect supersaturation. However, elasticity theory assumes that the point defect produces no shear and thus there is no work done by shear stress components of the dislocation over the strain of the defect $[4,5]$. This has been considered in previous work by $[6,7,8]$. In addition, purely elastic predictions of the stress field of Volterra dislocations will be inaccurate as the defect approaches the dislocation core. Moreover, it has been suggested by Kamiyama et. al. [9] that SIAs change orientation near the core of the edge dislocation in BCC metals. Therefore, investigations into the the final fate of point defects and defect clusters approaching the dislocation core, require methods based on atomistic simulation.

In the present paper, we present a molecular dynamics study of the interaction of self intcrstitial atoms (SIAs) and vacancics with edgc dislocations in iron crystals. The simulations are carried out with a many-body Finnis-Sinclair type interatomic potential with fixed boundary conditions. The initial atomic displacements are obtained from the anisotropic elasticity solution of the strain field of the dislocation. We obtain the value of the dislocation sink strength in the form of a temperature and angle dependent capture radius defined as the distance where the interaction energy between the dislocation and the defect is of the order of $k T$. Here, $k$ is Boltzmann's constant and $T$, the crystal temperature. These results can then be used directly as input data for mesoscopic theories of microstructure evolution under irradiation or kinetic Monte Carlo simulations. Recent work by Kamiyama et. al. [9] has also analyzed this problem using molecular dynamics. However, those calculations all assumed periodic boundary conditions along all crystal directions (owing to the interaction of the defect with dislocation images, the results may not be accurate) and discussed only a very small subset of possible interaction angles between the SIA and the dislocation.

Our results show that the non-isotropic nature of the strain field of the SIA in Fe induces a very strong interaction with the dislocation core even at positions where no interaction is predicted by simple elasticity considerations. We present results for the difference in capture radii of the vacancy and SIA in Fe and cast the results in terms of a dislocation sink strength as a function of temperature.

\section{Simulation Technique}

Molecular Dynamics (MD) with quasi-Newton quenching was used to obtain equilibrium atomic configuration of a crystal with an embedded edge dislocation and a point defect. In order to accelerate the attainment of equilibrium, atomic velocities were rescaled every timestep to fix the temperature of the crystal at a low value, typically $0.001 \mathrm{~K}$. All atoms were initially displaced according to values prescribed by the elastic anisotropic displacement field of a $1 / 2\langle 111\rangle\{110\}$ edge dislocation in Iron. The elastic anisotropic displacement field is calculated using the sextic formulation of Stroh [10]. Whereas atoms along the boundary 
of the simulation cell are kept fixed, periodic boundary conditions are imposed along the dislocation line.

Self Interstitial Atoms in BCC iron are introduced in the crystal as dumbbells with axes oriented along a $\langle 110\rangle$ direction. In the dumbbell configuration, the SIA shares a lattice site with a host atom. Vacancies are introduced in iron crystals by removing an atom in the host lattice.

The atoms in the crystal are then equilibrated using MD until the potential energy of the system reaches a steady value, accurate to $+/-0.01 \mathrm{eV}$. An MD timestep of $10^{-15}$ seconds is used. Typically, static equilibrium configurations are obtained after 10 picoseconds $\left(1 \mathrm{ps}=10^{-12} \mathrm{~s}\right)$. In subsequent runs, the point defect is placed successively closer to the dislocation and the corresponding equilibrium energy values are measured. Figure 1 shows the geometry of the calculation that will be discussed below.

The edge dislocation lies on a (110) plane and its Burgers vector is [111]. SIAs are introduced below the dislocation core at $\theta=0,225$ and 270 degrees, and vacancies above at $\theta=0,45$ and 90 degrees.

The interaction energy between the point defect and the dislocation is the difference between the energy value corresponding to a reference configuration in which the point defect is located infinitely far away from the dislocation and that corresponding to the defect located at a finite distance from the dislocation. The convergence of the calculations with respect to crystal size is checked by recomputing the interaction energy using simulations with crystals of larger sizes. Typically, we have found that simulation cells with $25,000-50,000$ atoms are adequate.

All simulations were carried out Finnis-Sinclair potential developed by Ackland et. al. [11] for iron. The potential predicts the most stable SIA configuration in $\mathrm{BCC}$ iron to be a $\langle 110\rangle$ dumbbell with formation energy, $4.87 \mathrm{eV}$. The formation energy for a

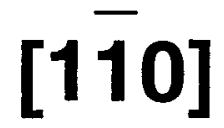

\}
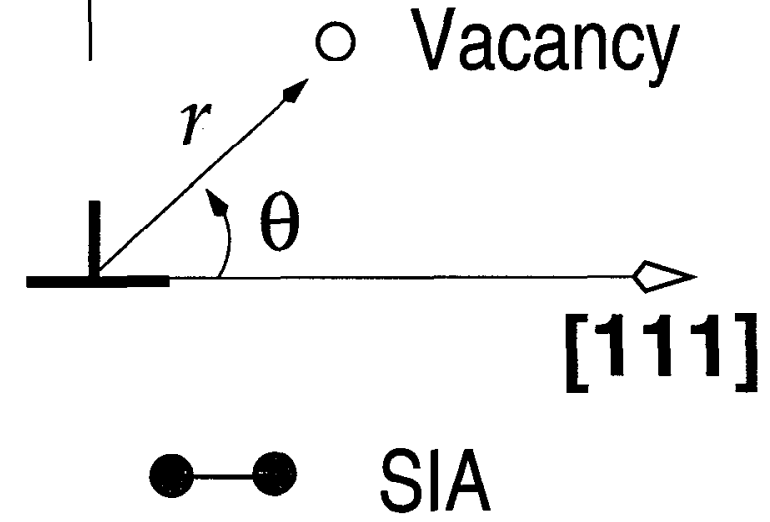

Figure 1: Schematic shows point defects located near an edge dislocation vacancy in iron is predicted to be $1.70 \mathrm{eV}$. Relaxation volumes of the point defects have also been calculated and found to have values of $1.76 \Omega_{0}$ and $-0.18 \Omega_{0}$ for the SIA and the vacancy, respectively.

\section{Results}

Figure 2 shows (a) a vacancy and (b,c) a SIA in two configurations, the ground state (110) dumbbell and the metastable (111) crowdion.

The color code refers to the strain field around the defect. The vacancy is observed to create an isotropic strain field, but the SIA does not. In particular, the strain field of the (111) crowdion is very anisotropic and focused along the (111) direction. It is interesting to note that recent calculations [12] have shown that the diffusion mechanism of the SIA in 


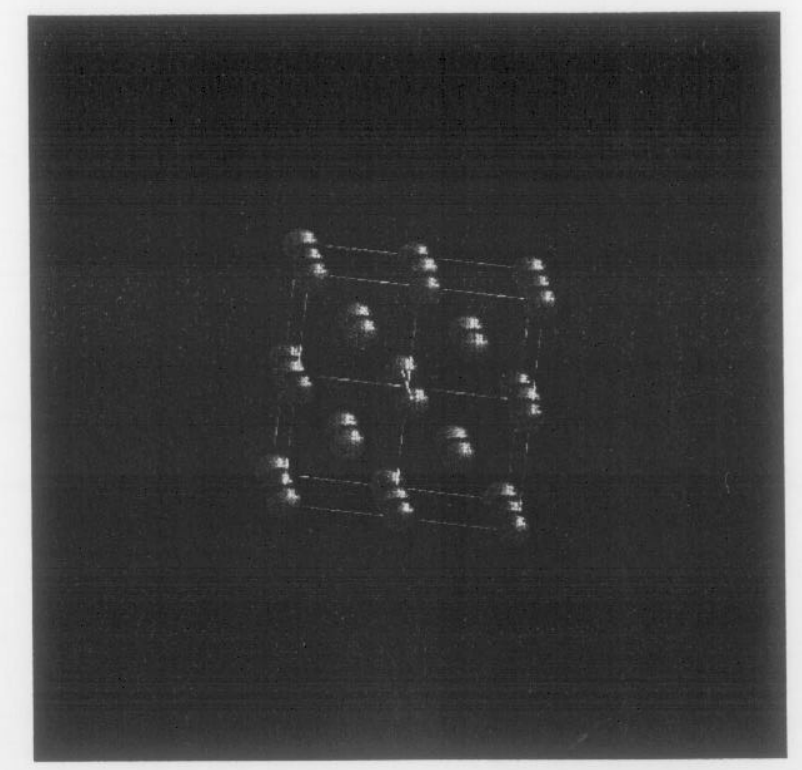

(a)

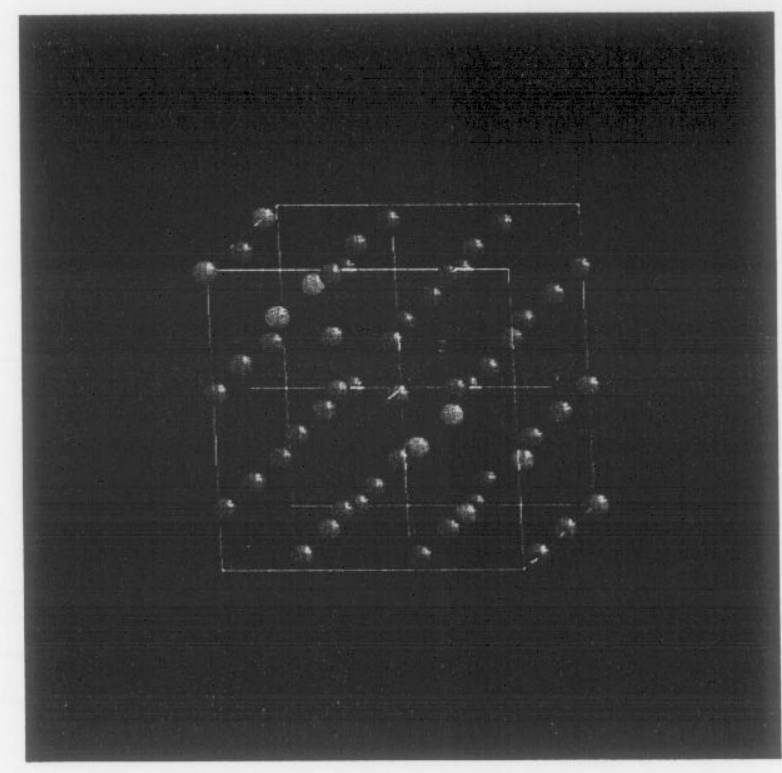

(b)

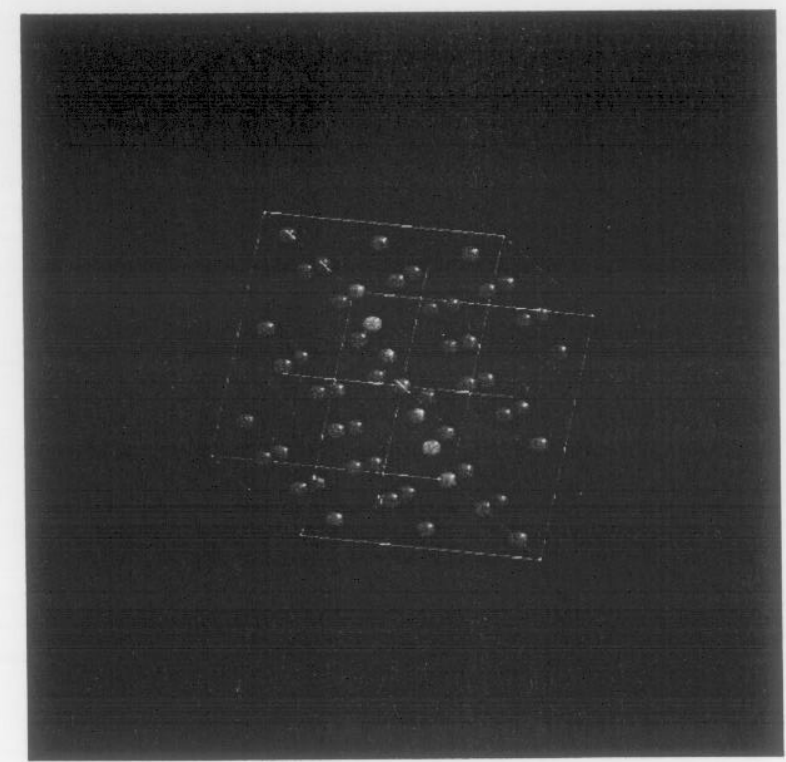

(c)

Figure 2: Atomic configurations corresponding to (a) a vacancy, (b) a $\langle 110\rangle$ dumbbell and (c) a $\langle 111\rangle$ crowdion SIA in iron. 
$\mathrm{Fe}$ involves a conversion from the (110) to the (111) configuration followed by the fast one dimensional migration of the (111)-oriented defect with a small activation energy of $0.04 \mathrm{eV}$. Thus, both SIA configurations participate in diffusion and must be considered when looking at the interaction between SIAs and dislocations.

Figure 3 shows the result of MD calculations of the formation (or interaction) energy, $E^{\text {int }}$, of a $\langle 110\rangle$ dumbbell SIA in $\mathrm{Fe}$ as a function of distance from the core of an edge dislocation.

These values are compared with the corresponding energy values obtained from the interaction of an isotropic defect with the elastic field of the edge dislocation. The elastic dislocation stress field used here is that due to the extended shear core of the edge dislocation. The distribution of shear in the core of an isolated edge dislocation was obtained from equilibrium atomic configurations predicted by MD, by examining the positions of atoms located on either side of the glide plane. The shear core of the perfect dislocation is then modeled as a distribution of discrete model Volterra dislocations, with Burgers vector equal to the incremental

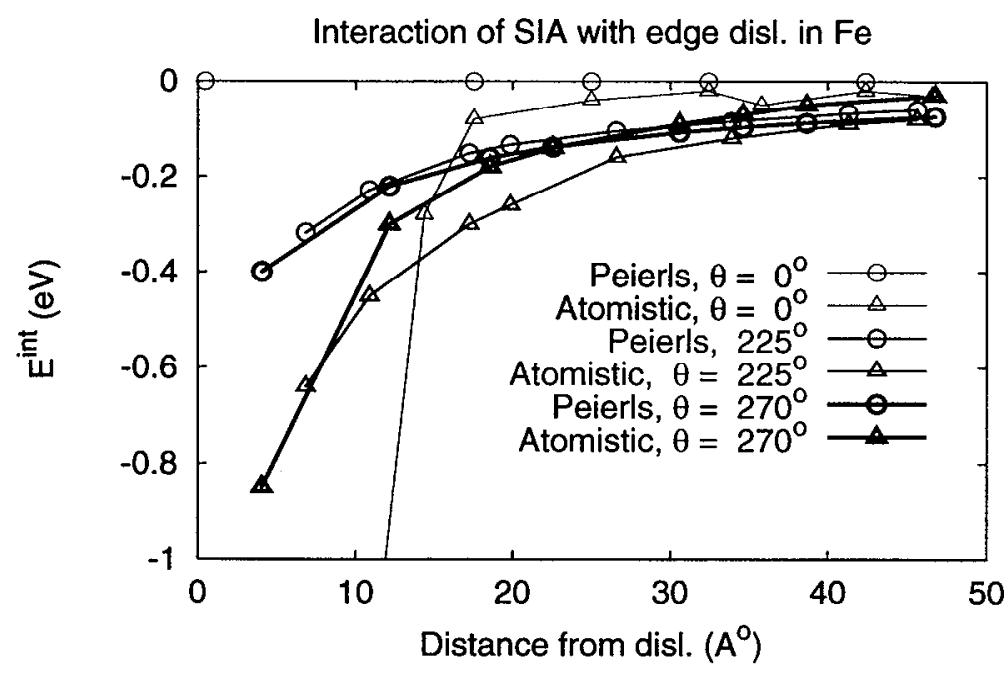

Figure 3: Comparison of Peierls and atomistic predictions for the interaction energy of an SIA with an edge dislocation in iron. shear at each model dislocation position. The total stress at the defect is found by linearly superposing the stress values of the individual model dislocations. The distribution of shear $\left(\Delta u_{x}\right)$ in the core is found to obey the functional form predicted by the Peierls model and it is

$$
\Delta u_{x}=b / \pi\left\{\pi / 2+\tan ^{-1}(x / w)\right\}
$$

with the core width parameter, $w=5.07 \AA$.

For a defect located immediately below the dislocation core $\left(\theta=270^{\circ}\right.$, where according simple elasticity arguments one would expect maximum interaction) the atomistic results show that as the defect approached the dislocation core, the formation energy is reduced by as much as $0.8 \mathrm{eV}$ at distances of the order of the burgers vector. This reduction is significantly larger than that obtained from the elasticity (Peierls) calculations described above. Similar differences between atomistic and Peierls results are observed for the $\theta=225^{\circ}$ case. Here, the formation energy is reduced by $0.7 \mathrm{eV}$ when the SIA approaches within a few Burgers vectors of the dislocation core.

When the defect is located on the glide plane of the dislocation, the results of the atomistic simulations deviate greatly from the elasticity calculations for the isotropic defect. 
In addition, the atomistic simulations show that the $\langle 110\rangle$ dumbbell is found to transform to a $\langle 111\rangle$ crowdion upon absorption by the dislocation.

Figure 4 shows compare the Peierls predictions with atomistic results for the interaction of a vacancy with the cdgc dislocation. The interaction energy between the edge dislocation and the vacancy is much smaller that for the SIA. However, even for the vacancy, there are departures from the Peierls predictions. In particular, the variations of interaction energy along $\theta=45^{\circ}$ and $90^{\circ}$ are observed to be very similar. Continuum formulations, on the other hand, predict the variations to be scaled by $\sin \theta$. The isotropic nature of the deformation field around vacancy is clearly reflected in the fact that no intcraction at $\theta=0$ is found in the atomistic simulations.

The results presented in figs. 3 and 4 can be used to obtain a capture radius. Following Heald and Speight [6], we define the capture radius as the distance at which $\Delta E$, i.e. the formation energy change of the point defect, becomes comparable to $k T$. The difference between the capture radius of the SIA and the vacancy is a measure of the sink strength of the dislocation. Figure 5a shows the capture radius for SIAs and vacancies when the defects are located immediately below and above the dislocation core, respectively. Figure $5 \mathrm{~b}$ shows the difference between these two numbers as a function of homologous temperature. For example, at reactor

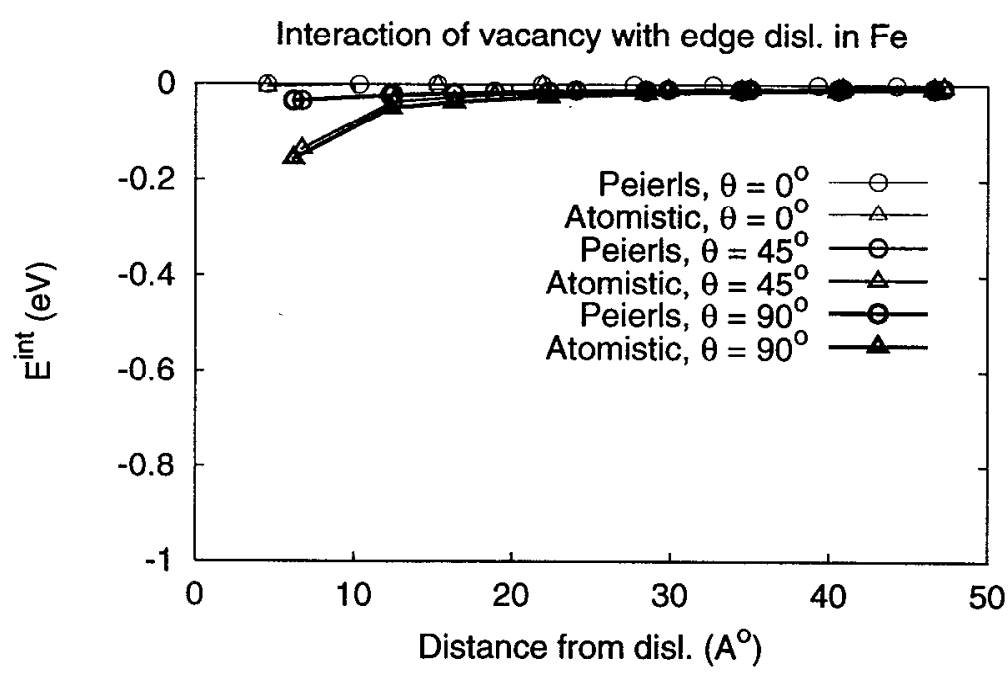

Figure 4: Comparison of Peierls and atomistic predictions for the interaction energy of a vacancy with an edge dislocation in iron. operating temperatures of $288^{\circ} \mathrm{C}$, the difference in the capture radii of the SI $\Lambda$ s and vacancies is $30 \AA$. In fact, it appears from these calculations that the capture radii of edge dislocations for point defects in BCC iron is rather small with values at typical operating temperatures of of the order of $10 \AA$ for vacancies and 40 Åfor SIAs.

\section{Summary and Conclusions}

The calculations presented above show that atomistic simulations are very valuable for obtaining detail information regarding the interaction of dislocations and point defects in irradiated materials. The results show that the strain field of the SIA in BCC iron has dilatational and shear components. This leads to a strong interaction with edge dislocations even when the defect is on the glide plane of the dislocation, where simple considerations based on elasticity and treatment of point defects as dilatational centers would predict no interaction. In fact, we have seen that on the glide planc, the SIA converts from a (110) 


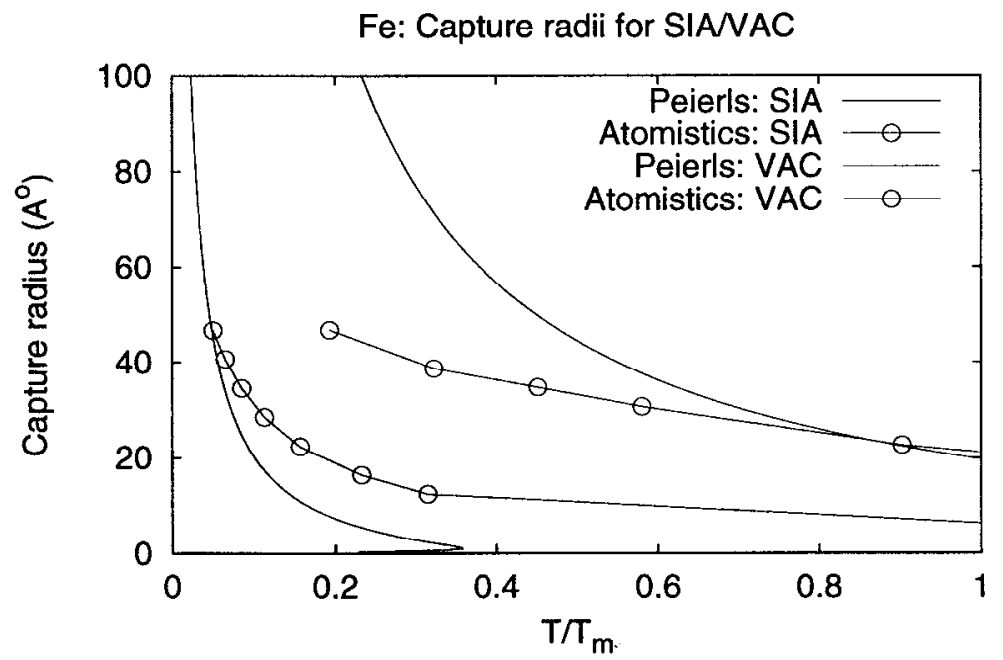

(a)

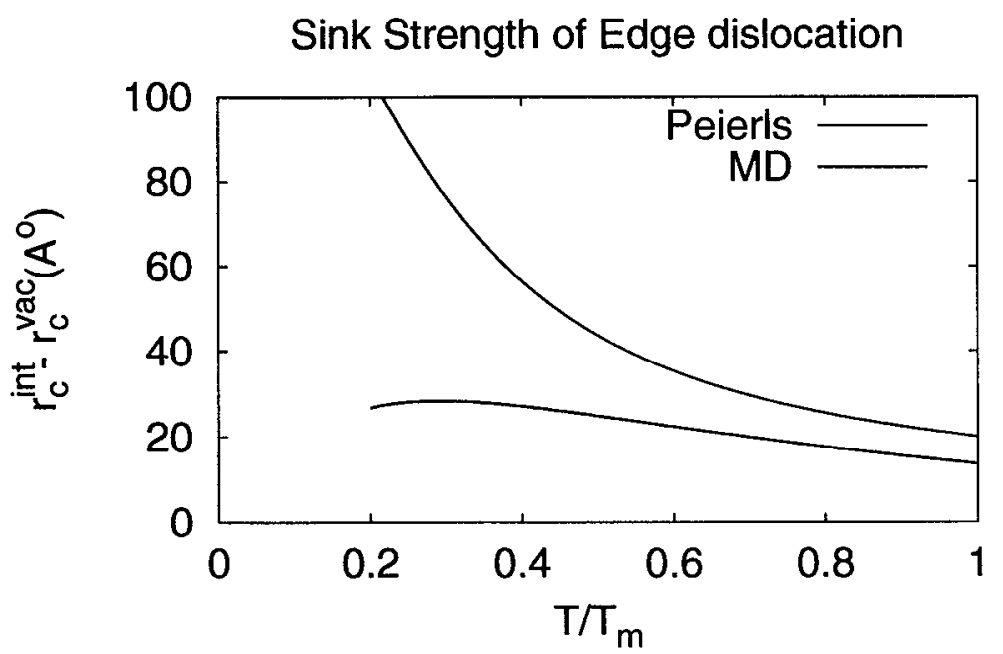

(b)

Figure 5: (a) Capture radii for SIA and vacancy in iron. (b) Comparison of sink strength predicted by atomistic and Peierls results. 
dumbbell configuration to the (111) crowdion and gets immediately absorbed in the core. For vacancies, the interaction is much weaker and the results are in much better agrecment with the prediction of the continuum theory.

The capture radii for the point defects obtained in these simulations are small at typical reactor operating temperatures and appear to indicate a small sink strength of edge dislocations in BCC iron. Calculations are under way to investigate the interaction between small glissile interstitial clusters and edge dislocations and betwecn all these defects and screw dislocations.

\section{References}

[1] A. D. Brailsford and R. Bullough, Philos. Trans. Roy. Soc., London 302, 87 (1981).

[2] L. K. Mansur, J. Nucl. Mater 216, 97 (1994).

[3] H. Trinkhaus, B. N. Singh, and A. J. E. Foreman, J. Nucl. Mater. 249, 91 (1997).

[4] J. P. Hirth and J. Lothe, Theory of Dislocations, McGraw-Hill, New York, 1968.

[5] D. Hull and D. J. Bacon, Introduction to Dislocations, Butterworth-Heinemann, Boston, Ma, 1981.

[6] P. T. Heald and M. V. Speight, Phil. Mag. 29, 1075 (1974).

[7] W. G. Wolfer and M. Ashkin, J. Appl. Phys. 47, 791 (1976).

[8] R. Bullough and J. R. Willis, Phil. Mag. 31, 855 (1975).

[9] H. Kamiyama, H. Rafii-Tabar, Y. Kawazoe, and H. Matsui, J. Nucl. Mater. 212-215, 231 (1994).

[10] A. N. Stroh, Phil. Mag. 3, 625 (1958).

[11] G. J. Ackland, D. J. Bacon, A. F. Calder, and T. Harry, Phil. Mag. A 75, 713 (1997).

[12] B. D. Wirth, G. R. Odette, and G. E. Lucas, J. Nucl. Mater. 244, 185 (1997). 